\title{
Imperforate Hymen about a Case
}

\author{
Babacar Biaye*, David Ngom, Aissatou Mbodji, Astou Coly Niassy Diallo, Aminata Niass, \\ Abdoul Aziz Diouf, Omar Gassama, Mamadou Lamine Cissé, Alassane Diouf, Jean Charles Moreau
}

Gynecological and Obstetric Clinic, Aristide Le Dantec Hospital of Dakar, Dakar, Senegal

Email: *drbabacarbiaye@yahoo.fr

How to cite this paper: Biaye, B., Ngom, D., Mbodji, A., Diallo, A.C.N., Niass, A., Diouf, A.A., Gassama, O., Cissé, M.L., Diouf, A. and Moreau, J.C. (2019) Imperforate Hymen about a Case. Open Journal of Obstetrics and Gynecology, 9, 474-478. https://doi.org/10.4236/ojog.2019.94046

Received: February 24, 2019

Accepted: April 9, 2019

Published: April 12, 2019

Copyright $\odot 2019$ by author(s) and Scientific Research Publishing Inc. This work is licensed under the Creative Commons Attribution International License (CC BY 4.0).

http://creativecommons.org/licenses/by/4.0/

\begin{abstract}
Imperforate hymen is a congenital obstructive abnormality of the female genital tract and its incidence is estimated at $1 / 2000$ female births. The diagnosis may go unnoticed during the examination of the new born in the birth room. Most often, this malformation is discovered at puberty. Treatment of Imperforate hymen is hymenotomy or hymenectomy. Different types of incisions are mentioned in the literature. We have reported the case of a 14-year-old girl with hematocolpos and hematometra on hymenal impforforate. In cultures and religions where the loss of the hymen is a social problem among unmarried girls, a medical certificate must be given to the patient.
\end{abstract}

\section{Keywords}

Imperforate Hymen, Hematocolpos, Hymenotomy

\section{Introduction}

Imperforate hymen is a rare malformation and its incidence is estimated at $1 / 2000$ female births [1]. It is most often diagnosed at puberty with the constitution of a hematocolpos by vaginal retention of the first menstruation. Early diagnosis and management are necessary to avoid complications [1] [2]. Treatment is a simple surgical procedure with a few types of incisions on the imperforate hymenal membrane. As it is evidence of virginity, an intact hymen is important in some cultures and religions. Since patients and families are afraid of losing their virginity after surgery, the surgeon must choose the best-controlled technique and the one that provides the best natural intact annular hymenal architecture if possible under these circumstances.

We report the case of a hymenal imperforation of a 14-year-old girl.

\section{Observation}

This is a 14-year-old girl with no specific history who consulted for cyclical pel- 
vic pain with cramps associated with primary amenorrhea.

The clinical examination found a hypogastric mass and a vaginal imperforation (Figure 1).

The ultrasound performed, found a hematocolpos and a hematometra of great abundance measuring $18 \times 9 \mathrm{~cm}$ without associated urinary abnormalities (Figure 2).

Management consisted of a hymenotomy with a cross incision on the membrane followed by an evacuation of $600 \mathrm{ml}$ of menstrual blood.

We excised flaps and performed hemostasis points around the orifice (Figure 3). Thus no operative incident was observed.

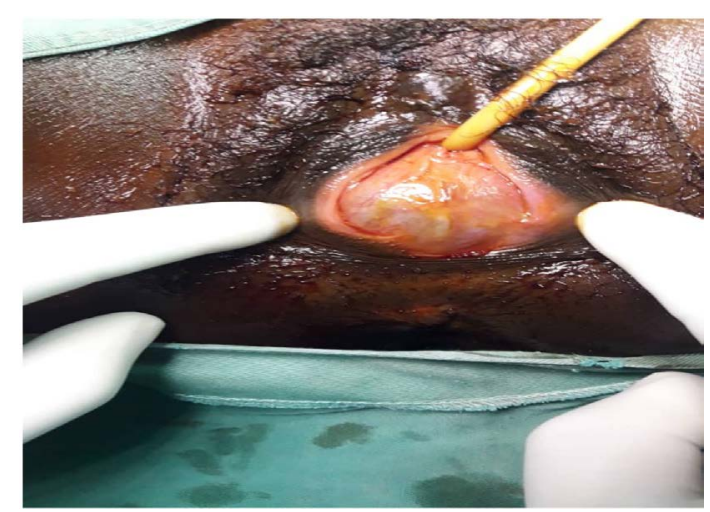

Figure 1. Imperforate hymen to clinical examination.

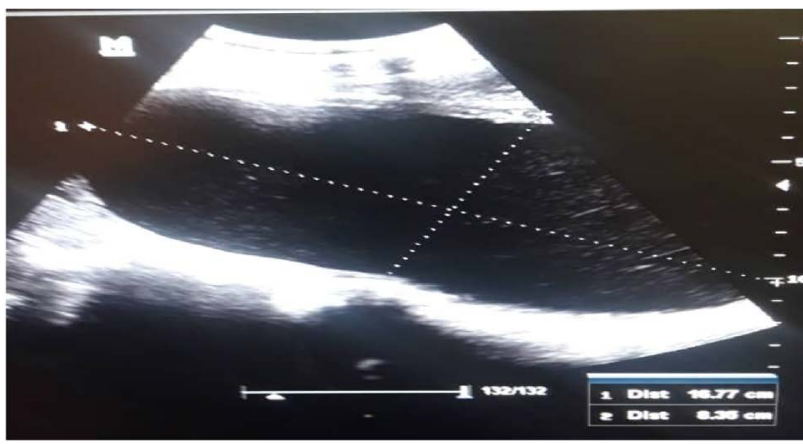

Figure 3. Hematometra on ultrasound.

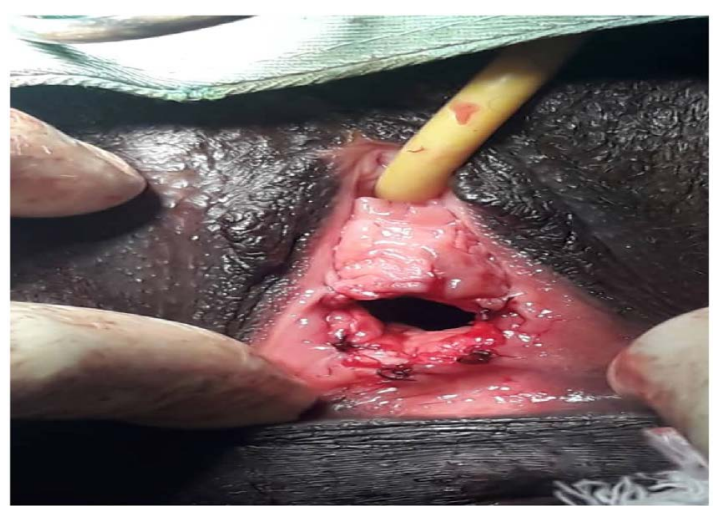

Figure 3. Postoperative appearanceof the vaginal opening. 


\section{Discussion}

Imperforate Hymen is a rare entity, but it is the most common genital malformation. Its incidence is estimated at 1/2000 female births [1].

In the embryological period, the lateral portion of the hymen originates from a fold of urogenital sinus at the union of Mùller's duct, whereas in its posterior part, it originates from the cells of the urogenital sinus externally and from Mùller's duct internally. Usually in the eighth week of gestation, it partially ruptures in the inferior part of Mùller's duct, remaining as a fold of mucous membrane around the entrance of the vagina. Failure of these events results in persistence of the septum, which can be diagnosed as imperforate hymen clinically. As it will cause vaginal outflow obstruction, there may be significant accumulation of cervical and vaginal secretions [3]. Imperforate hymen is not usually associated with any other Mullerian abnormalities.

The majority of cases reported in the literature are sporadic; however, some familial cases have been described suggesting a probable genetic predisposition [4]. No familial concept of hymenal imperforation has been reported in this patient.

The diagnosis of imperforate hymen is possible in utero in front of the ultrasonographic finding of a hydrometocolpos [5]. In utero diagnosis also has the advantage of looking for associated renal malformations. This diagnosis can be made by systematic screening at birth but also before a hydrometrocolpos during the genital crisis of the new-born female. Most often, this malformation is discovered at puberty. The diagnosis must be suspected in front of a girl presenting with primary amenorrhea with normally developed secondary sexual characteristics.

Patients usually consult for recurrent pelvic pain secondary to the accumulation of blood in the vagina or hematocolpos or even in the uterus (hematometry) [6]. Such a clinical picture was found in our patient. Retrograde menstrual flow may alter the fallopian tubes or lead to endometriotic lesions that may impede fertility later. However, this possibility is rare if the diagnosis is made early and fertility is generally maintained [7].

The cyclic nature of painful seizures may be lacking due to the usual irregularity of the menstrual cycle during the peripubertal period. The pains can be misleading, pseudo-appendicular and induce interventions "in excess" for suspicion of acute appendicitis. Hematocolpos can compress the urethra and cause dysuria, complete bladder retention, or even bilateral uretero-hydronephrosis [1] [5], Einsenberg reported through a series of 44 Hematocoplos, 7 cases of bladder retention. Constipation is the same compression mechanism [8].

The clinical diagnosis of this malformation is most often easy. Examination of the abdomen reveals an oval suprapubic swelling, with a large, superior end, with regular contours, of fluctuating or renitent consistency, sensitive, matte to percussion, and plunging down behind the pubic symphysis. The inspection of the vulva makes it possible to recognize the imperforation by showing a bluish 
translucent membrane projecting between the labia minora. The digital rectal examination perceives a median, anterior swelling of fluid consistency, which resembles near the anal sphincter [9].

When in doubt, an ultrasound can be performed that will objectify an anechoic vaginal and intra-uterine retro-vesical image. This ultrasound examination can make it possible to perform a pelvic malformation assessment, particularly in search of a urinary malformation. In our patient, the ultrasound showed no associated malformation [10]. If the diagnosis of imperforate hymen is not absolutely certain based on physical examination or pelvic ultrasonographic findings, an MRI should be obtained to clearly define the anatomy prior to any planned surgical procedure. This MRI seems to be the best examination for malformation assessment. However, our patient was not able to do this examination because of lack of financial means.

The management is aimed at re-establishing vaginal outflow and mainly consists of surgical hymenotomy or hymenectomy [11]. Different types of incisions are mentioned in the literature. Some prefer simple vertical, T-shaped, cruciform, X-shaped and cyclical incisions may be used [11]. X-shaped incision has the advantage of reduced risk of injury to the urethra-which should be stented during the procedure [12]. Pressure on the uterus in order to expel more blood is discouraged as it can lead to retrograde flow through the tubes causing endometriosis and tubal adhesions. However others prefer simple central excision of the hymen making an annular-intact hymen using a foley catheter for 2 weeks in order to prevent restenosis. This last technique as similar restenosis risks, so incision type depends on patient's desire or surgeon's preferance. In cultures where the destruction of the hymen is a social problem in unmarried girls, it is important to preserve the annular structure of the hymen in gynecological practice.

Our team having never practiced the simple hymenotomy with conservation of the hymen, we prefer to make hymenotomy with a cross incision on the membrane associating the hymenectomy.

Hymenectomy and hymenotomy with a two week indwelling catheter have also been reported [11]. The outcome is good and the recurrences are rare.

In our society where the Muslim religion is the most dominant, questions of virginity occupy an important place in the project of marriage. It is in this context that we issued a medical certificate attesting a surgical loss of virginity in this teenager as a justification. However there is no law requiring the prescription of this medical certificate.

\section{Conclusion}

Imperforate hymen is an entity of female genital malformations. It is an often benign affection, of favorable evolution, if it is diagnosed and treated early. Conversely, his lack of knowledge exposes him to serious complications. Ultrasonography remains the test of choice to confirm the diagnosis, and allows to identify a possible upstream reverberation and associated urogenital abnormali- 
ties. Routine screening at birth and early treatment are the best guarantors for preventing the complications of this pathology. The treatment is exclusively surgical and several procedures have been described.

\section{Conflicts of Interest}

The authors declare no conflicts of interest regarding the publication of this paper.

\section{References}

[1] Posner, J.C. and Spandorfer, P.R. (2005) Early Detection of Imperforate Hymen Prevents Morbidity from Delays in Diagnosis. Pediatrics, 115, 1008-1012. https://doi.org/10.1542/peds.2004-0183

[2] Heger, A.H., Ticson, L., Guerra, L., et al. (2002) Appearance of the Genitalia in Girls Selected for Nonabuse: Review of Hymenal Morphology and Nonspecific Findings. Journal of Pediatric and Adolescent Gynecology, 15, 27-35. https://doi.org/10.1016/S1083-3188(01)00136-X

[3] Battran, V.C. (1983) Mullerian Anomalies and Their Management. Fertility and Sterility, 40, 159-163. https://doi.org/10.1016/S0015-0282(16)47230-4

[4] Erdemoglu, E., Kolusarı, A., Şahin, G.H. and Kamacı, M. (2007) Familyal imperfore hymen. Journal of the Turkish German Gynecology Association, 8, 88-89.

[5] Chircop, R. (2003) A Case of Retention of Urine and Haematocolpometra. European Journal of Emergency Medicine, 10, 244-245. https://doi.org/10.1097/00063110-200309000-00019

[6] Letts, M. and Haasbeek, J. (1990) Hematocolpos as a Case of Back Pain in Premenarchal Adolescents. Journal of Pediatric Orthopaedics, 10, 731-732. https://doi.org/10.1097/01241398-199011000-00005

[7] Joki-Erkkilä, M.M. and Heinonen, P.K. (2003) Presenting and Long-Term Clinical Implications and Fecundity in Females with Obstructing Vaginal Malformations. Journal of Pediatric and Adolescent Gynecology, 16, 307-312. https://doi.org/10.1016/S1083-3188(03)00157-8

[8] Einsenberg, E. and Faber, M. (1982) Complete Duplication of the Uterus and Cervix with Unilateraly Imperforate Vagina. Obstetrics \& Gynecology, 60, 259-262.

[9] Ercan, C.M. (2011) Imperforate Hymen Causing Hematocolpos and Acute Urinary Retention in an Adolescent Girl. Taiwanese Journal of Obstetrics \& Gynecology, 50, 118-120. https://doi.org/10.1016/j.tjog.2011.01.005

[10] Robberecht, E., Smets, A., Wincker, M.V. and Delens, F. (1996) Radiological Case of the Month Hematocolpos Due to Imperforate Hymen. Archives of Pediatrics and Adolescent Medicine, 150, 993-994. https://doi.org/10.1001/archpedi.1996.02170340107020

[11] Chelli, D., Kehila, M., Sfar, E., Zouaoui, B., Chelli, H. and Chanoufi, B. (2008) Imperforate Hymen: Can It Be Treated without Damaging the Hymenal Structure? Sante, 18, 83-87.

[12] Acar, A., Balci, O., Karatayli, R., Capar, M. and Colakoglu, M. (2007) The Treatment of 65 Women with Imperforate Hymen by a Central Incision and Application of Foley Catheter. BJOG, 114, 1376-1379. https://doi.org/10.1111/j.1471-0528.2007.01446.x 